\title{
THEOREMS CONNECTED WITH MACLAURIN'S TEST FOR THE CONVERGENCE OF SERIES
}

\author{
$B y$ G. H. HARDY. \\ [Received and Read, April 28th, 1910.]
}

1. In a paper recently published in these Proceedings, ${ }^{*}$ Dr. Bromwich gave a very interesting extension of the theorem 'usually but inaccurately ascribed to Cauchy) that, when $f(x)$ is a positive decreasing function of $x$, the series

$$
\stackrel{\infty}{\Sigma} f(n)
$$

and the integral

$$
\int^{\infty} f(x) d x
$$

converge or diverge together. Dr. Bromwich proved that, if

(i) $f(x)$ is positive and tends steadily to zero;

(ii) $\phi(x)$ is positive and tends steadily to infinity,

(iii) $\phi^{\prime}(x)$ tends steadily to zero,

(iv) the integral $\int^{\infty} f(x) \phi^{\prime}(x) d x$ is convergent, then

$$
\int^{x} F(x) d x-\stackrel{[x]}{\Sigma} F(n),
$$

where $F(x)=f(x) e^{i \phi(x)}$ and $[X]$ denotes the integral part of $X$, tends to a finite limit as $X \rightarrow \infty$.

Roughly, it may be said that what Dr. Bromwich proved amounted to this, that the relations between (1) and (2), as regards convergence or divergence, established by Maclaurin and Cauchy when $f(x)$ is positive and decreasing, still subsist when $f(x)$ is multiplied by an oscillatory factor of the type

$$
\cos \phi(x),
$$

provided $\phi(x)$ tends to infinity more slowly than $x$. 
1910.] Maclaurin's test For the convergence of SERies.

2. Dr. Bromwich's theorem may be generalised and its proof simplified as follows :-

Theовем 1.-If (i) $F(x)$ possesses a continuous derivative $F^{\prime}(x)$, (ii) $F(x) \rightarrow 0$ as $x \rightarrow \infty$, (iii) the integral

$$
\int^{\infty}\left|F^{\prime \prime}(x)\right| d x
$$

is convergent, then

$$
\int_{0}^{X} F(x) d x-\sum_{1}^{[X]} F(n)
$$

tends to a finite limit, viz.,

as $X \rightarrow \infty . *$

$$
J_{1}=-\int_{0}^{\infty}(x-[x]) F^{\prime \prime}(x) d x
$$

Let $\quad j_{\nu}=\int_{\nu-1}^{\nu} F(x) d x-F(\nu)=\int_{\nu-1}^{\nu}\{F(x)-F(\nu)\} \frac{d}{d x}(x-\nu+1) d x$

$$
=-\int_{\nu-1}^{\nu}(x-[x]) F^{\prime}(x) d x \text {. }
$$

Then

$$
\left|j_{\nu}\right| \leqslant \int_{\nu-1}^{\nu}\left|F^{\prime}(x)\right| d x
$$

Hence $\Sigma j_{v}$ is convergent (absolutely) and the result follows immediately, since

$$
\int_{[x]}^{x} F(x) d x \rightarrow 0 .
$$

3. In the case considered by Dr. Bromwich, we have

$$
\left.F^{\prime}(x)=\vdots f^{\prime}(x)+i f(x) \phi^{\prime}(x)\right\} e^{i \phi(x)},
$$

and the convergence of $\int^{\infty}\left|F^{\prime}(x)\right| d x$ follows immediately from that of

$$
\int^{\infty} f^{\prime}(x) d x, \quad \int^{\infty} f(x) \phi^{\prime}(x) d x \text {. }
$$

Dr. Bromwich points out to me that the last inequality of $\$ 2$ may be established with equal simplicity by the method of argument adopted by bimself (loc. cit., p. 329). In fact

$$
j_{v}=\int_{r-1}^{\nu}\left\lceil F(x)-F(\nu)_{i}^{\prime} d x=-\int_{r-1}^{\nu} d x \int_{r}^{v} F^{\prime}(t) d t,\right.
$$

and so

$$
\left|j_{\nu}\right| \leqslant \int_{\nu-1}^{\nu}\left|F^{\prime}(t)\right| d t
$$

So far as applications are concerned, there is practically nothing to choose

* It is not difficult to establish a similar theorem relating to double series and integrals. 
between Dr. Bromwich's result and mine. For the sake of completeness I give an example in which my result applies and his does not. It is obtained by taking

$$
F(x)=x^{-\imath} \exp \left\{i x^{b} \cos (\log x)\right\},
$$

where $0<b<a<1$.

4. Either theorem enables us, as Dr. Bromwich shows, to determine very simply the behaviour, from the point of view of convergence, of many interesting series, such as $\Sigma(\log n)^{p} n^{-1-a i}$. But neither theorem is sufficiently general to deal with many other simple and interesting cases that naturally present themselves.

Consider, for example, the series

$$
\Sigma \frac{e^{i n^{n}}}{n^{b}}
$$

where $0<a<1, b>0$. The integral

$$
\int^{\infty} \frac{e^{i x^{n}}}{x^{b}} d x
$$

is convergent if $a+b>1$, and it is natural to suppose that the same is the case with the series: and this is in fact true. But the conditions of Theorem 1 are satisfied only if $b>a$.

It is therefore desirable to investigate more general theorems.

5. It will easily be verified that, if we transform the expression $j_{\nu}$ of $\S 2$ by a further integration by parts, we obtain*

$$
\int_{\nu-1}^{\nu}\left\{f(x)+\frac{1}{2} f^{\prime}(x)\right\} d x-f(\nu)=\frac{1}{2} \int_{\nu-1}^{\nu}\left(y^{2}-y\right) f^{\prime \prime}(x) d x,
$$

or, say,

$$
j_{\nu}^{(2)}=\frac{1}{2} \int_{\nu-1}^{\nu}\left(y^{2}-y\right) f^{\prime \prime}(x) d x
$$

where

$$
y=x-[x] \text {. }
$$

And generally, if we denote by $\phi_{k}(x)$ the Bernoullian polynomial of degree $k$, so that $\dagger$

$\phi_{1}=x, \quad \phi_{2}=x(x-1), \quad \phi_{3}=x(x-1)\left(x-\frac{1}{2}\right), \quad \phi_{4}=x^{2}(x-1)^{2}$,

* In what follows I revert to the notation $f(x), f(n)$ instead of $F(x), F(n)$.

$\dagger$ Bromwich. Infinite Series, p. 236. 
1910.] MacLAURIN'S TES'S For THE CONVERGENCE OF SERIES.

we have

$$
\text { (3) } \begin{aligned}
j_{\nu}^{(2 k)}= & \int_{\nu-1}^{\nu}\left\{f(x)+\frac{1}{2} f^{\prime}(x)+\frac{B_{1}}{2 !} f^{\prime \prime}(x)-\right. \\
& \quad+\frac{B_{2}}{4 !} f^{(4)}(x)+\ldots \\
= & -\frac{1}{(2 k+1) !} \int_{\nu-1}^{\nu} \phi_{2 k+1}(y) f^{(2 k+1)}(x) d x \\
= & \left.\frac{1}{(2 k+2) !} \int_{\nu-1}^{\nu} \phi_{2 k+2}(y) f^{(2 k)}(x)\right\} d x-f(\nu)
\end{aligned}
$$

These formulæ are, in fact, merely a slight transformation of one of the standard forms of the "Euler-Maclaurin Sum Formula." *

From (8) we at once deduce

ThEOREM 2.-If either of the integrals

$$
\int^{\infty}\left|f^{(2 k+1)}(x)\right| d x, \quad \int^{\infty}\left|f^{(2 l+2)}(x)\right| d x
$$

is convergent, then

$$
\int_{0}^{[X]}\left\{f(x)+\frac{1}{2} f^{\prime}(x)+\frac{B_{1}}{2 !} f^{\prime \prime}(x)-\ldots\right\} d x-\sum_{1}^{[X]} f(\nu)
$$

tends, as $X \rightarrow \infty$, to a finite limit, viz., one of the integrals

$$
\begin{aligned}
& J_{2 k+1}=-\frac{1}{(2 k+1) !} \int_{0}^{\infty} \phi_{2 k+1}(x-[x]) f^{(2 k+1)}(x) d x, \\
& J_{2 k+2}=\frac{1}{(2 k+2) !} \int_{0}^{\infty} \phi_{2 k+2}(x-[x]) f^{(2 k+2)}(x) d x .
\end{aligned}
$$

If also $f(x)$ and its first $2 k$ derivatives tend to zero, as $x \rightarrow \infty$, then

$$
\int_{0}^{X} f(x) d x-\sum_{1}^{[X]} f(\nu)
$$

tends, as $X \rightarrow \infty$, to the limit

$$
\frac{1}{2} f(0)+\frac{B_{1}}{2 !} f^{\prime}(0)-\frac{B_{2}}{4 !} f^{(3)}(0)+\ldots+\frac{(-1)^{k-1} B_{k}}{2 k !} f^{(2 k-1)}(0)+J_{l},
$$

where $l=2 k+1$ or $l=2 k+2$, as the case may be.

We have, in fact, merely to observe that the maximum of $\left|\phi_{2 k+1}(y)\right|$ or $\left|\phi_{2 k+2}(y)\right|$ depends on $k$ only and not on $[X]$.

\footnotetext{
- Bromwich, Infinite Series, p. 239.
}

SER. 2. VOL. 9. NO. 1075. 
I have not thought it worth while to encumber this theorem (or the others which follow) with explicit statements as regards the continuity of such derivatives of $f(x)$ as figure in them. All such derivatives are, of course, supposed continuous : only conditions relating to their behsviour as $x \rightarrow \infty$ are in any way relevant to the problem. Sometimes, however, these conditions may be broken for $x=0$ or some other value of $x$. Then the formula may need modification, but the application of the theorem to questions of convergence is not affected.

6. If

$$
f(x)=x^{-b} e^{i x^{a}},
$$

where $0<a<1$, it is easy to see that

$$
f^{(s)}(x) \sim(i a)^{s} x^{s(a-1)-b} e^{i x^{n}},
$$

which yields an absolutely convergent integral if $s(a-1)-b<-1$ or

$$
s>(1-b) /(1-a) \text {. }
$$

It is always possible to choose $s$ so as to satisfy this condition : hence the series and integral

$$
\int x^{-h} e^{i x^{n}} d x, \quad \Sigma n^{-b} e^{i n^{a}} \quad(0<a<1)
$$

in all cases converge or oscillate together, i.e., converge if, and only if, $a+b>1$.

If $b>a$ is implied by $a+b>1$, i.e., if $a \leqslant \frac{1}{2}$, but not if $a>\frac{1}{2}$, this result may be inferred from Theorem 1 .

7. It is naturally suggested by the preceding results that it should be possible to prove theorems of a similar character, but relating to series and integrals that are only summable and not convergent. The integral

$$
\int_{0}^{\infty} f(x) d x
$$

is said to be summable $(C r)$, to sum $S$, if

$$
\frac{r !}{x^{r}}\left(\int_{0}^{x} d t\right)^{r+1} f(t) \rightarrow S
$$

as $x \rightarrow \infty . *$

This definition is strictly analogous to Cesàro's definition of the sum

- Some properties of integrals which are summable $(C 1)$ are proved by Hardy, Quarterly Journal, Vol. xxxv, p. 22 ; Moore, Trans. Amer. Math. Soc., Vol. viII, p. 299 ; Bromwich, Math. Annalen, Bd. Lxv, S. 350 . The above definition is an immediate generalisation of that adopted by these writers. 
$s$ of an oscillatory series $\Sigma a_{v}$, which may be put in the form

$$
\frac{r !}{n^{r}}\left(\sum_{\nu=1}^{n}\right)^{r+1} a_{\nu} \rightarrow s
$$

Now, by a well known formula,*

$$
\left(\int_{0}^{x} d t\right)^{r+1} f(t)=\frac{1}{r !} \int_{0}^{x}(x-t)^{r} f(t) d t
$$

Hence our definition is equivalent to

$$
\int_{0}^{x}\left(1-\frac{t}{x}\right)^{r} f(t) d t \rightarrow S
$$

or

$$
\int_{0}^{1} x f(x u)(1-u)^{r} d u \rightarrow S .
$$

And, when either of these forms is adopted, there is no longer any reason why $r$ should be restricted to be an integer; we may suppose that $r$ has any real value greater than -1 .

Suppose, e.g., that

$$
f(x)=x^{p} e^{a i x},
$$

where $a$ is real and not zero. Then the integral on the left-hand side of (7) is

$$
x^{p+1} \int_{0}^{1} u^{p}(1-u)^{r} e^{n i x u} d u .
$$

Now it is known that, when $|x|$ is large,

$$
\int_{0}^{1} u^{p}(1-u)^{r} e^{a i x u} d u=\frac{\Gamma(p+1)}{(-a i x)^{p+1}}(1+\epsilon)+\frac{\Gamma(r+1)}{(a i x)^{r+1}} e^{a i x}\left(1+\epsilon^{\prime}\right),
$$

where $\epsilon, \epsilon^{\prime}$ are small of the order $1 / x$, and the many valued functions in the denominators have their values fixed by conventions explained precisely in the papers referred to in the foot-note below. $\dagger$

It follows at once that

$$
\int_{0}^{\infty} x^{n} e^{a i x} d x
$$

is summable $(\mathrm{Cr})$, and has the sum

$$
\Gamma(p+1) /(-a i)^{p+1}
$$

if, and only if, $r>p$.

- See, e.g., Jordan, Cours d'Analyse, t. III, p. 59.

$\dagger$ Jacobsthal, Math. Annalen, Bd. r.v, S. 129 ; Barnes, Trans. Camb. Phil. Soc., Vol.xx, p. 253 ; Hardy, Proc. London Math. Soc., Ser. 2, Vol. 3, p. 401. 
8. Now M. Marcel Riesz has replaced* Cesàro's definition by

$$
\sum_{\nu=1}^{n}\left(1-\frac{\nu}{n}\right)^{r} a_{\nu} \rightarrow s
$$

a definition precisely analogous in form to that adopted in equation (7) above for the value of the summable integral. This formal analogy renders it convenient to adopt this definition in the analysis which follows.

Before proceeding further, I may remark that I shall consider explicitly only the case in which $r$ is a positive integer. This is the really interesting case. But the definition (8), like (7), applies equally well for non-integral values of $r$, and, in a good deal of the succeeding analysis, the restriction that $r$ is integral is in no way necessary.

9. M. Riesz has indicated an important generalisation of the definition expressed by (8). Let $\lambda(x)$ be any function of $x$ which tends steadily to infinity with $x$. Then we miy define the sum $s$ of the series $\Sigma a_{n}$ by the relation

$$
\sum_{\nu=1}^{n}\left\{1-\frac{\lambda(\nu)}{\lambda(n)}\right\}^{r} a_{\nu} \rightarrow s
$$

which reduces to $(8)$ if $\lambda(x)=x$. If this limit exists, we shall say that the series $\Sigma a_{n}$ is summable $(R v)$ with sum-function $\lambda(n)$. When $r=1$ this definition reduces to

or to

$$
\begin{gathered}
s_{n}-\frac{1}{\lambda(n)} \sum_{\nu=1}^{n} \lambda(\nu) a_{\nu} \rightarrow s \\
\frac{\mu_{2} s_{1}+\mu_{8} s_{2}+\ldots+\mu_{n} s_{n-1}}{\lambda(n)} \rightarrow s,
\end{gathered}
$$

where $\mu_{\nu}=\lambda(\nu)-\lambda(\nu-1)$; which is the definition that I considered in a recent paper in these Proceedings. $t$

The corresponding definition for the integral is obviously

$$
\left.\int_{0}^{x} i^{i} 1-\frac{\lambda(t)}{\lambda(x)}\right)^{r} f(t) d t \rightarrow S
$$

- Comptes Rendus, July 5th, 1909. M. Riesz has established (as is easy cnough when $r$ is integral) the substantial equivalence of the two definitions.

+ Prnc. Isondon Math. Soc., Ser. 2. Vol. 8, p. 301. 
If this limit exists, I shall say that the integral

$$
\int_{0}^{\infty} f(x) d x
$$

is summable (Rr), with sum-function $\lambda(x)$, to sum $S$.

If we put

$$
\lambda(t)=T, \quad \lambda(x)=X,
$$

and denote by $\bar{\lambda}$ the function inverse to $\lambda$, we see that (10) is equivalent to

$$
\int_{0}^{x}\left(1-\frac{T}{X}\right)^{r} f\{\bar{\lambda}(T)\} \bar{\lambda}^{\prime}(T) d T .
$$

That is to say,

$$
\int^{\infty} f(x) d x
$$

is summable $(R r)$, with sum-function $\lambda(x)$, if, and only if,

$$
\left.\int^{\infty} f: \bar{\lambda}(x)\right\} \bar{\lambda}^{\prime}(x) d x
$$

is summable $(R r)$, with sum-function $x$. The adoption of Riesz's general definition* instead of Cesàro's is, in the case of integrals, substantially equivalent to a change in the independent variable.

Thus it is easy to prove that

$$
\int^{\infty}(\log x)^{p} x^{-1+a i} d x
$$

is not summable $(\mathrm{Cr})$ for any value of $r$. The substitution $x=e^{y}$, however, leads to the integral

$$
\int^{\infty} y^{\prime \prime} e^{a i ! \prime} d y
$$

which is summable $(C r)$ for $r>p$. Hence (11) is summable $(R r)$, with sun-function $\log x$, for $r>p$. We shall see in a moment that the same is true of the series

$$
\Sigma(\log n)^{p} n^{-1+a i} \text {. }
$$

10. We are now in a position to establish the following theorem :-

Theorem 3.-If $f(x)$ is subject to the conditions of Theorem 1, viz., that $f(x) \rightarrow 0$ and

$$
\int^{\infty}\left|f^{\prime}(x)\right| d x
$$

* I call the definition (10) Riesz's because he has doubtless used it as well as the definition (9). It would indeed be a plausible conjecture that M. Riesz was led to the definitions (8) and (9) througb some consideration of integrals. 
is convergent, and $\lambda(x)$ is any function of $x$ which has a continuous derivative and tends steadily to infinity with $x$, then

$$
\Sigma_{n}=\int_{0}^{n}\left\{1-\frac{\lambda(t)}{\lambda(n)}\right)^{r} f(t) d t-\sum_{1}^{n}\left\{1-\frac{\lambda(\nu)}{\lambda(n)}\right\}^{r} f(\nu)
$$

tends, as $n \rightarrow \infty$, to the finite limit $J_{1}$ of Theorem 1 , so that the summability of the series $\Sigma f(\nu)$ follows from that of the integral, the definitions of summability being Riesz's definitions of the r-th order, with sum-functions $\lambda(x), \lambda(n)$.

Let

$$
\psi(t)=\left\{1-\frac{\lambda(t)}{\lambda(n)}\right\}^{r} f(t) .
$$

Then

$$
\Sigma_{n}=\sum_{1}^{n} j_{v}
$$

where $j_{\nu}=\int_{\nu-1}^{\nu} \psi(t) d t-\psi(\nu)=-\int_{\nu-1}^{\nu}(t-[t]) \psi^{\prime}(t) d t=u_{\nu}+v_{\nu}+w_{\nu}$, $u_{v}, v_{v}, v_{v}$ being defined by the equations

$$
\begin{aligned}
& u_{\nu}=-\int_{\nu-1}^{\nu}(t-[t]) f^{\prime}(t) d t, \\
& v_{\nu}=\int_{\nu-1}^{\nu}(t-[t])\left(1-\left\{1-\frac{\lambda(t)\}^{r}}{\lambda(n)}\right) f^{\prime}(t) d t,\right. \\
& w_{\nu}=\frac{r}{\lambda(n)} \int_{\nu-1}^{\nu}\left\{1-\frac{\lambda(t)}{\lambda(n)}\right\}^{r-1} \lambda^{\prime}(t) f(t) d t .
\end{aligned}
$$

In the first place, $\quad\left|u_{v}\right|<\int_{\nu-1}^{\nu}\left|f^{\prime}(t)\right| d t$,

so that $\Sigma u_{\nu}$ is convergent (absolutely). In the second place,

$$
\left|w_{\nu}\right|<\frac{r}{\lambda(n)} \int_{\nu-1}^{\nu} \lambda^{\prime}(t)|f(t)| d t .
$$

But it is easy to prove that $\phi(t) \rightarrow 0$ involves

$$
\frac{1}{\lambda(n)} \int_{0}^{n} \phi(t) \lambda^{\prime}(t) d t \rightarrow 0: *
$$

* This is an immediate generalisation from the well known case in which $\lambda(n)=n$. Choose $N$ so that $|\phi|<\epsilon$ for $x \geqslant N$. Then

$$
\frac{1}{\lambda(n)}\left|\int_{0}^{n} \phi \lambda^{\prime} d t<\frac{-1}{\lambda(n)} \int_{0}^{N}\right| \phi \lambda^{\prime}\left|d t+\frac{\epsilon}{\lambda(n)} \int_{N}^{n} \lambda^{\prime}(t) d t<\frac{1}{\lambda(n)} \int_{0}^{N}\right| \psi \lambda^{\prime} \mid d t+\epsilon<2 \epsilon,
$$

if $n$ is large enough. 
and so

$$
\sum_{1}^{n} w_{v} \rightarrow 0
$$

Finally,

$$
\left|\sum_{1}^{n} v_{\nu}\right|<\int_{0}^{n}\left(1-1-\frac{\lambda(t)}{\lambda(n)} !^{r}\right)\left|f^{\prime}(t)\right| d t .
$$

Let

$$
\int_{t}^{\infty}\left|f^{\prime}(u)\right| d u=\Phi(t)
$$

Then, integrating by parts,

$$
\begin{aligned}
\left|\sum_{1}^{n} v_{\nu}\right|<-\Phi(n) & +\left(1-\left\{1-\frac{\lambda(0)}{\lambda(n)}\right\}^{r}\right) \Phi(0) \\
& -\frac{r}{\lambda(n)} \int_{0}^{n}\left\{1-\frac{\lambda(t)}{\lambda(n)}\right\}^{r-1} \lambda^{\prime}(t) \Phi(t) d t .
\end{aligned}
$$

The first two terms evidently tend to zero as $n \rightarrow \infty$. The last is numerically less than

$$
\frac{r}{\lambda(n)} \int_{0}^{n} \lambda^{\prime}(t)|\Phi(t)| d t
$$

and so also tends to zero.

It follows that $\quad \Sigma_{u} \rightarrow \sum_{1}^{\infty} \iota_{\nu}=-\int_{0}^{*}(t-[t]) f^{\prime}(t) d t$,

and the proof of Theorem 3 is accordingly completed.

11. A simple example of the theoren just proved is afforded by the case in which

$$
f(x)=(\log x)^{p} d^{-1+a i} .
$$

Here

$$
f^{7}(x) \sim(a i-1)(\log x)^{p} x^{-2+a i},
$$

and the conditions of the theorem are certainly satistied. It follows from $\$ 9$ that the series

$$
\sum(\log n)^{\mu} n^{-i+a i}
$$

is summable $(R r)$, with sum function $\log n$, if, and only if, $r>p$.

12. The question now arises as to whether we can prove a still more general theorem related to Theorem 3 as Theorem 2 is related to Theorem 1. 
I shall content myself with proving this in two special cases, viz., (1) when

$$
\lambda(x)=x
$$

(so that we are using definitions substantially equivalent to Cesàro's), and (2) when $\lambda(x)$ is arbitrary and $k=0$. 'The absolutely general case appears to require rather elaborate preliminaries.

13. The formula (3) of $\S 5$, applied to the function

$$
\psi(t)=\left(1-\frac{t}{n}\right)^{r} f(t)
$$

gires

$$
\begin{aligned}
\int_{\nu-1}^{\nu}\left(\psi(t)+\frac{1}{2} \psi^{\prime}(t)+\frac{B_{1}}{2 !} \psi^{\prime \prime}(t)\right. & \left.-\ldots+\frac{(-1)^{k-1} B_{k}}{2 k !} \psi^{(2 k)}(t)\right\} d t-\psi(\nu) \\
& =\frac{1}{(2 k+2) !} \int_{\nu-1}^{\nu} \phi_{2 k+2}(t-[t]) \psi^{(2 k+2)}(t) d t
\end{aligned}
$$

Suppose now that, as in Theorem 2,

$$
\int^{\infty}\left|f^{(2 k+2)}(t)\right| d t
$$

and is convergent, and also that $f^{(2 k+1)}(t) \rightarrow 0$. Then we can prove that

$$
\sum_{1}^{n} \int_{\nu-1}^{\nu} \phi_{2 k+2}(t-[t]) \psi^{(2 k+2)}(t) d t \rightarrow \int_{0}^{\infty} \phi_{2 k+2}(t-[t]) f^{(2 k+2)}(t) d t .
$$

We have, in fact,

$$
\begin{aligned}
\psi^{(2 k+2)}(t)=f^{(2 k+2)}(t) & -\left\{1-\left(1-\frac{t}{n}\right)^{r} ; f^{(2 k+2)}(t)\right. \\
+ & \sum_{s=1}^{\sigma}(-1)^{s} s !\left(\begin{array}{c}
2 k+2 \\
s
\end{array}\right)\left(\begin{array}{l}
r \\
s
\end{array}\right) n^{-s}\left(1-\frac{t}{n}\right)^{r-s} f^{(2 k+2-s)}(t),
\end{aligned}
$$

where $\sigma$ is the lesser of the numbers $2 k+2$ and $r$.

We may therefore write

$$
\int_{\nu-1}^{\nu} \phi_{2 k \div 2}(t-[t]) \psi^{(2 k+2)}(t) d t=u_{\nu}+v_{\nu}+\sum_{s=1}^{\sigma} w_{\nu}^{(s)}
$$


where $\quad u_{\nu}=\int_{\nu-1}^{\nu} \phi_{2 k+2}(t-[t]) f^{(2 k+2)}(t) d t$,

$$
\begin{aligned}
& v_{\nu}=-\int_{\nu-1}^{\nu} \phi_{2 k+2}(t-[t])\left\{1-\left(1-\frac{t}{n}\right)_{j}^{r} f^{(2 k+2)}(t) d t,\right. \\
& w_{\nu}^{(v)}=C_{s} n^{-s} \int_{\nu-1}^{\nu} \phi_{2 k+2}(t-[t])\left(1-\frac{t}{n}\right)^{r-s} f^{(2 k+2-s)}(t) d t,
\end{aligned}
$$

$C_{s}$ being a number dependent only on $k, r, s$, and not upon $\nu$ or $n$.

Since

$$
\left|u_{\nu}\right|<M \int_{\nu-1}^{\nu}\left|f^{(2 k+2)}(t)\right| d t
$$

where $M$ is the maximum of $\phi_{2 k+2}(y)$ for $0<y<1$, it follows that $\Sigma u_{v}$ is convergent (absolutely).

$$
\text { Again, } \quad\left|\sum_{1}^{n} v_{\nu}\right|<M \int_{0}^{n}\left\{1-\left(1-\frac{t}{n}\right)^{r}\right\}\left|f^{(2 k+2)}(t)\right| d t .
$$

Let

$$
\Phi(t)=\int_{t}^{\infty}\left|f^{(2 k+2)}(u)\right| d u .
$$

Then $\int_{0}^{n}\left\{1-\left(1-\frac{t}{n}\right)^{r}\right\}\left|f^{(2 k+2)}(t)\right| d t=-\Phi(n)+\frac{r}{n} \int_{0}^{n}\left(1-\frac{t}{n}\right)^{r-1} \Phi(t) d t$.

The first term on the right-hand side has the limit zero. The second is less than

$$
\frac{r}{n} \int_{0}^{n} \Phi(t) d t
$$

and so also tends to zero. It follows that

$$
\sum_{1}^{n} v_{\nu} \rightarrow 0 \text {. }
$$

Finally,

$$
\left|\sum_{1}^{n} w_{v}^{(s)}\right|<K n^{-s} \int_{0}^{n}\left|f^{(2 k+2-s)}(t)\right| d t,
$$

where $K$ is independent of $n$. I shall prove in the next section that the expression on the right-hand side tends to zero as $n \rightarrow \infty$, for $s=1,2, \ldots, \sigma$. It will then follow that

$$
\sum_{1}^{n} w_{\nu}^{(s)} \rightarrow 0 \quad(s=1,2, \ldots, \sigma),
$$

and from (16) and (17) will follow the truth of the assertion made at the beginning of this section. 
14. Since $f^{(2 k+1)}(t) \rightarrow 0$, it follows at once that

$$
\frac{1}{t} \int_{0}^{t}\left|f^{(2 k+1)}(u)\right| d u \rightarrow 0,
$$

as $t \rightarrow \infty$. Hence

$$
\left|f^{(2 k)}(t)\right| \leqslant\left|f^{(2 k)}(0)\right|+\int_{0}^{t}\left|f^{(2 k+1)}(u)\right| d u=t \epsilon_{\iota},
$$

where $\epsilon_{t} \rightarrow 0$. Hence

$$
\frac{1}{t^{2}} \int_{0}^{t}\left|f^{(2 k)}(u)\right| d u \rightarrow 0,
$$

and from this we deduce as above that

$$
\left|f^{(2 k-1)}(t)\right|=t^{2} \epsilon_{t},
$$

where $\epsilon_{t} \rightarrow 0$. It is evident that a mere repetition of this argument is sufficient to prove what we require.

It is therefore proved that the sum from $v=1$ to $\nu=n$ of the righthand side of the equation (15) tends, as $n \rightarrow \infty$, to the limit

$$
J_{2 k++2}=\frac{1}{(2 k+2) !} \int_{0}^{x} \phi_{2 k+2}(t-[t]) f^{(2 k+2)}(t) d t .
$$

15. We have now to consider the left-hand side of (15), which is

$$
\begin{aligned}
\Sigma_{n}+\frac{1}{2}\{\psi(n)-\psi(0)\} & +\frac{B_{1}}{2 !}\left\{\psi^{\prime}(n)-\psi^{\prime}(0) !-\frac{B_{2}}{4 !}\left\{\psi^{(3)}(n)-\psi^{(3)}(0)\right\}+\ldots\right. \\
& +\frac{(-1)^{k-1} B_{k}}{2 k !}\left\{\psi^{(2 k-1)}(n)-\psi^{(2 k-1)}(0)\right\}
\end{aligned}
$$

where

$$
\Sigma_{n}=\int_{0}^{n}\left(1-\frac{t}{n}\right)^{r} f(t) d t-\sum_{1}^{n}\left(1-\frac{\nu}{n}\right)^{r} f(\nu) .
$$

Now

$$
\psi^{(s)}(t)=\sum_{\kappa=0}^{\lambda}(-1)^{\kappa} \kappa !\left(\begin{array}{l}
s \\
\kappa
\end{array}\right)\left(\begin{array}{l}
r \\
\kappa
\end{array}\right) n^{-\kappa}\left(1-\frac{t}{n}\right)^{r-\kappa} f^{(s-\kappa)}(t),
$$

where $\lambda$ is the lesser of the numbers $r, s$. From this it follows at once that

$$
\psi^{(s)}(0)=f^{(s)}(0)+\epsilon_{n},
$$


where $\epsilon_{n} \rightarrow 0$ as $n \rightarrow \infty$. Also

$$
\psi^{(s)}(n)=0 \quad(r>s)
$$

since, if $r>s$, every term in $\psi^{(s)}(n)$ contains a factor which vanishes for $t=n$. On the other hand, if $r \leqslant s$, there is one term in $\psi^{(s)}(n)$ which does not vanish for $t=n$, viz., that for which $\kappa=r$, and

$$
\psi^{(s)}(n)=(-1)^{r} r !\left(\begin{array}{l}
s \\
r
\end{array}\right) n^{-r} f^{(s-r)}(n) \quad(r \leqslant s) .
$$

From (18), (19), and (20) it follows that the left-hand side of (15) may be expressed in the form

$$
\begin{aligned}
& \Sigma_{n}+\epsilon_{n}-\frac{1}{2} f(0)-\frac{B_{1}}{2 !} f^{\prime}(0)+\frac{B_{2}}{4 !} f^{(3)}(0)-\ldots-\frac{(-1)^{k-1} B_{k}}{2 k !} f^{(2 k-1)}(0)+\chi(n), \\
& \text { where } \quad \chi(n)=(-1)^{r} r ! n^{-r} \Sigma \frac{(-1)^{p-1} B_{p}}{2 p !}\left(\begin{array}{c}
2 p-1 \\
r
\end{array}\right) f^{(2 p-1-r)}(n),
\end{aligned}
$$

the summation extending to all the values of $p$ (if any) which satisfy the inequalities

$$
r \leqslant 2 p-1 \leqslant 2 k-1 \text {. }
$$

We therefore assume, in addition to the conditions already imposed upon $f$, that

$$
x^{-r} f^{(2 p-1-r)}(x) \rightarrow 0,
$$

for $r \leqslant 2 p-1 \leqslant 2 k-1$. Thus, if $r=1$, we must have

$$
f(x) / x \rightarrow 0, f^{\prime \prime}(x) / x \rightarrow 0, \ldots, f^{(2 k-2)}(x) / x \rightarrow 0 ;
$$

if $r=2 k-1$, we must have

$$
f(x) / x^{2 k-1} \rightarrow 0 ;
$$

while, if $r \geqslant 2 k$, no conditions of this type are needed. If, e.g., $k=1$, the only case in which one of these conditions is required is when $r=1$, the condition then being

$$
f(x) / x \rightarrow 0 .
$$

16. We have thus proved-

Theorem 4.- - If

$$
\begin{aligned}
& \text { (i) } \int^{\infty}\left|f^{(2 k+2)}(x)\right| d x \text { is convergent, } \\
& \text { (ii) } f^{2 k+1}(x) \rightarrow 0, \\
& \text { (iii) } f^{(2 p-1-r)}(x) / x^{r} \rightarrow 0
\end{aligned}
$$


for such values of $p$, if any, as satisfy the inequalities

$$
r \leqslant 2 p-1 \leqslant 2 k-1
$$

then

$$
\int_{0}^{n}\left(1-\frac{t}{n}\right)^{r} f(t) d t-\sum_{1}^{n}\left(1-\frac{\nu}{n}\right)^{r} f(\nu)
$$

tends, as $n \rightarrow \infty$, to the limit

$$
\begin{aligned}
\frac{1}{2} f(0) & +\frac{B_{1}}{2 !} f^{\prime}(0)-\frac{B_{2}}{4 !} f^{(3)}(0)+\ldots \\
& +\frac{(-1)^{k-1} B_{k}}{2 k !} f^{(2 k-1)}(0)+\frac{1}{(2 k+2) !} \int_{0}^{\infty} \phi_{2 k+2}(t-[t]) f^{(2 k+2)}(t) d t,
\end{aligned}
$$

so that the summability $(R v)$, with sum-function n, of the series $\Sigma f(n)$ follows from that of the integral $\int f(x) d x$.

In the simplest case, that of $k=0$, the theorem takes the form

ThEOREM 5.-If (i) $\int^{\infty}\left|f^{\prime \prime}(x)\right| d x$ is convergent, (ii) $f^{\prime}(x) \rightarrow 0$, then

$$
\int_{0}^{n}\left(1-\frac{t}{n}\right)^{r} f(t) d t-\sum_{1}^{n}\left(1-\frac{\nu}{n}\right)^{r} f(\nu) \rightarrow \frac{1}{2} f(0)+\frac{1}{2 !} \int_{0}^{\infty} \phi_{2}(t-[t]) f^{\prime \prime}(t) d t,
$$

if $r>0$; and if $r=0$, the result is still true, provided only

$$
f(x) \rightarrow 0 \text {. }
$$

17. Let us consider, for example, the case in which

$$
f(x)=x^{-b} e^{i x^{*}} .(0<a<1) .
$$

Then

$$
f^{(s)}(x) \sim(i a)^{s} x^{s(a-1)-b} e^{i x^{a}},
$$

which tends to zero, if $s(a-1)-b<0$, and possesses an absolutely convergent integral, if $s(a-1)-b<-1$. This last condition will be satisfied for $s=2 k+2$, if

$$
2 k+2>(1-b) /(1-a) .
$$

This is certainly so for sufficiently large values of $k$. 
The condition that $f^{(2 k+1)}(x) \rightarrow 0$ requires

$$
2 k+1>-b /(1-a) ;
$$

and, as $1 /(1-a)>1$, this is a consequence of the former condition.

The condition

$$
f^{(2 p-1-r)}(x) / x^{r} \rightarrow 0,
$$

reduces to

$$
(2 p-1-r)(a-1)-b-r<0 \text {. }
$$

This is least likely to be satisfied when $p$ has its smallest possible value, viz., that given by $r=2 p-1$. It then reduces to

$$
b+r>0 \text {. }
$$

But the condition $b+r>0$ is certainly a necessary condition for the summability of

$$
\Sigma n^{-b} e^{i n^{a}}
$$

by Cesàro's $r$-th mean.* Hence there is no loss of generality involved in this set of conditions.

We can now apply Theorem 4, and we see that the problem of the summability of the series is reduced to the corresponding problem for the integral

$$
\int x^{-b} e^{i x^{a}} d x \text {. }
$$

We have therefore only to determine whether

$$
\int_{0}^{n}\left(1-\frac{t}{n}\right)^{r} \frac{e^{i^{a}}}{t^{b}} d t
$$

tends to a limit as $n \rightarrow \infty$. Putting

$$
t=n u^{1 / a}=(N u)^{1^{\prime} a},
$$

we obtain $\quad \frac{1}{a} N^{(1-b) / a} \int_{0}^{1}\left(1-u^{1 / a}\right)^{r} u^{[(1-b) / a]-1} e^{i N u} d u$.

Now it may easily be proved, by a slight modification of the argument used in establishing the asymptotic formula quoted in $\S 7$, that

$$
\int_{0}^{1}\left(1-u^{1 / a}\right)^{r} u^{[(1-b) / a]-1} e^{i N u} d u=\frac{\Gamma\{(1-b) / a\}}{(-i N)^{(1-b) / a}}(1+\epsilon)+\frac{\Gamma(r+1)}{a^{r}(i N)^{r+1}} e^{i N}\left(1+\epsilon^{\prime}\right),
$$

* Bromwich, Infinite Series, p. 318. 
where $\epsilon$ and $\epsilon^{\prime}$ are of order $1 / N$ when $N$ is large. It follows that the integral

$$
\int_{0}^{\infty} \frac{e^{i u^{n}}}{t^{b}} d t
$$

is summable $(\mathrm{Cr})$, and has the sum

$$
\frac{1}{a} \Gamma\left(\frac{1-b}{a}\right) /(-i)^{(1-b) / a},
$$

if, and only if, $r+1>(1-b) / a$; i.e., if

$$
b+(r+1) a>1 .^{*}
$$

And so the same is true of the series: that is to say, the series and integral

$$
\sum n^{-b} e^{i n^{a}}, \quad \int^{\infty} x^{-b} e^{i x^{a}} d x \quad(0<a<1)
$$

are both convergent if $a+b>1$, both summable $(R 1)$ if $2 a+b>1$, both summable (R2) if $3 a+b>1$, and so on, with sum function $n$.

18. The last question which arises is as to whether there is an analogue of Theorem 4, when Riesz's more general definition is adopted. The answer is, of course, in the affirmative; but, for the reason stated in $\$ 12$, I content myself with considering the simplest case, the analogue of Theorem 5.

THEorey 6. - If (i) $\int^{\infty}\left|f^{\prime \prime}(x)\right| d x$ is convergent, (ii) $f^{\prime}(x) \rightarrow 0$, (iii) $\lambda(x)$ is a steadily increasing function of $x$, tending to infinity with $x$, and possessing a continuous derivative, (iv) $\left\{\lambda^{\prime}(x) / \lambda(x)\right\} f(x) \rightarrow 0$, then

$$
\int_{0}^{n}\left\{1-\frac{\lambda(t)}{\lambda(x)}\right\}^{r} f(t) d t-\sum_{1}^{n}\left\{1-\frac{\lambda(\nu)}{\lambda(n)}\right\}^{r} f(\nu)
$$

tends, as $n \rightarrow \infty$, to the limit

$$
\frac{1}{3} f(0)+\frac{1}{2} \int_{0}^{\infty} \phi_{2}(t-[t]) f^{\prime \prime}(t) d t,
$$

if $r>0$; and the conclusion remains valid for $r=0$, provided $f(x) \rightarrow 0$.

* Of course we suppose $b<1$ in order that the integral should converge at the lower limit: this is quite irrelevant to the main issue. If $b>1$, the integral is absolutely convergent as regards the upper limit. 
We have, in fact, if $\Sigma_{n}$ denotes the expression whose limit we are considering,

$$
\Sigma_{n}+\frac{1}{2}\{\psi(n)-\psi(0)\}=\frac{1}{2 !} \int_{0}^{n} \phi_{2}(t-[t]) \psi^{\prime \prime}(t) d t,
$$

where

$$
\psi(t)=\left\{1-\frac{\lambda(t)}{\lambda(n)}\right\}^{r} f(t)
$$

Clearly $\psi(n)=0$, unless $r=0$, in which case we impose the additional condition that

$$
\psi(x)=f(x) \rightarrow 0 .
$$

Again,

$$
\begin{aligned}
\psi^{\prime \prime}(t)=\left\{1-\frac{\lambda(t)}{\lambda(n)}\right\}^{r} f^{\prime \prime}(t) & -\frac{2 r \lambda^{\prime}(t)}{\lambda(n)}\left\{1-\frac{\lambda(t)}{\lambda(n)}\right\}^{r-1} f^{\prime}(t) \\
& +\frac{r(r-1)\left\{\lambda^{\prime}(t)\right\}^{2}}{\{\lambda(n)\}^{2}}\left\{1-\frac{\lambda(t)}{\lambda(n)}\right\}^{r-2} f(t) \\
& \left.\left.-\frac{r \lambda^{\prime \prime}(t)}{\lambda(n)}\right\}_{i} 1-\frac{\lambda(t))}{\lambda(n)}\right\}^{r-1} f(t) .
\end{aligned}
$$

The reader who has followed the arguments of the preceding paragraphs with care (especially those of $\$ \$ 10,13$ ) will easily convince himself that everything reduces to proving that $f^{\prime}(x) \rightarrow 0$ involves

$$
\begin{aligned}
& \text { (a) } \frac{1}{\lambda(x)} \int_{0}^{x} \lambda^{\prime}(t) f^{\prime}(t) d t \rightarrow 0, \\
& \text { (b) } \frac{1}{i \lambda(x)_{i}^{\prime 2}} \int_{0}^{x}\left\{\lambda^{\prime}(t)\right\}^{2} f(t) d t \rightarrow 0, \\
& \text { (c) } \frac{1}{\lambda(x)} \int_{0}^{x} \lambda^{\prime \prime}(t) f(t) d t \rightarrow 0 .
\end{aligned}
$$

Of these relations the first has already been proved. Again,

$$
\frac{1}{\lambda(x)} \int_{0}^{x} \lambda^{\prime \prime}(t) f(t) d t=\frac{\lambda^{\prime}(x)}{\lambda(x)} f(x)-\frac{\lambda^{\prime}(0)}{\lambda\left(x^{\prime}\right)} f(0)-\frac{1}{\lambda(x)} \int_{0}^{x} \lambda^{\prime}(t) f^{\prime}(t) d t,
$$

and so also tends to zero if the condition

$$
\frac{\lambda^{\prime}(x)}{\lambda(x)} f(x) \rightarrow 0
$$


is satisfied, as we have supposed. Also, if this condition is satisfied, we can find $X$, so that

$$
|f(x)|<\epsilon \lambda(x) / \lambda^{\prime}(x) \quad(x \geqslant X) .
$$

Then $\frac{1}{\{\lambda(x)\}^{2}}\left|\int_{0}^{x}\left\{\lambda^{\prime}(t)\right\}^{2} f(t) d t\right|<\frac{1}{\{\lambda(x)\}^{2}} \int_{0}^{X}\left\{\lambda^{\prime}(t)\right\}^{2}|f(t)| d t$

$$
+\frac{\epsilon}{\{\lambda(x)\}^{2}} \int_{X}^{x} \lambda(t) \lambda^{\prime}(t) d t
$$

The second term is less than $\frac{1}{2} \epsilon$; and by choosing $x$ large enough, when $X$ is fixed, we can ensure that the first term is also less than $\frac{1}{2} \epsilon$. The relations $(a),(b),(c)$ are accordingly established and Theorem 6 is proved. 